\title{
Tandem-X bistatic polarimetric mode for maritime applications
}

\author{
Ferdinando Nunziata \\ and Maurizio Migliaccio \\ Università di Napoli Parthenope \\ Dipartimento di Ingegneria \\ Centro Direzionale, isola C4 - Napoli
}

\author{
Domenico Velotto \\ German Aerospace Center (DLR), Germany \\ Earth Observation Center, Remote Sensing Technology Institute \\ Bremen, Germany.
}

\begin{abstract}
In this study, TanDEM-X full-polarimetric bistatic and monostatic imagery are exploited for maritime applications purposes. The Mueller matrix and the phase difference between co-polarized channels (CPD) are exploited to deal with both the monostatic and the bistatic scenes. Subtle differences are found between the Mueller matrix collected in bistatic/monostatic mode; while similar performance is achieved when the CPD is exploited.
\end{abstract}

\section{INTRODUCTION}

TanDEM-X opens a new era in space borne radar remote sensing since it is the first bistatic Synthetic Aperture Radar (SAR) mission. Although, primary mission objective is the generation of a consistent global digital elevation model (DEM) with an unprecedented accuracy, TanDEM-X provides a highly reconfigurable platform for the demonstration of new SAR techniques and applications. Bistatic SAR imaging provides additional observable for the extraction of important scene and target parameters. TanDEM-X allows for the simultaneous acquisition of bistatic and monostatic fullpolarimetric images in a single data take to obtain a highly informative set of multi-angle observations. In this study, this unprecedented amount of information will be exploited for maritime observation purposes, i.e. to observe ships and coastal areas. Experiments will be carried on actual TanDEM$\mathrm{X}$ data collected in Gulf of Naples, Italy.

\section{Methodology}

A full-polarimetric SAR measures the scattering matrix associated to the observed scene:

$$
\boldsymbol{E}^{\mathrm{s}}=\frac{e^{-\mathrm{j} k r}}{r} \mathbf{S} \boldsymbol{E}^{\mathrm{s}}
$$

where $\mathrm{j}$ is the imaginary unit, $k$ is the electromagnetic wavenumber, $r$ is the distance between the SAR antenna and the center of the imaged scene, and $\boldsymbol{E}^{\mathrm{s}}$ and $\boldsymbol{E}^{\mathrm{i}}$ are the complex Jones vectors describing the scattered and incident field, respectively. $\mathbf{S}$ is a $2 \times 2$ complex matrix that, when the linear $\{h, v\}$ basis is adopted, is given by:

$$
\mathbf{S}=\left(\begin{array}{cc}
S_{\mathrm{hh}} & S_{\mathrm{hv}} \\
S_{\mathrm{vh}} & S_{\mathrm{vv}}
\end{array}\right)
$$

whose complex elements $S_{p q}$, with $p, q \in\{h, v\}$, are termed as scattering amplitudes. It is important to underline that, for a given frequency and viewing geometry, the scattering matrix depends only on the scattering properties of the observed scene [1]. Note that in the bistatic case, $S_{h v} \neq S_{v h}$, while in the backscattering case and under backscatter alignment convention cross-polarized scattering amplitudes are equal. Eq. (2) represents a first-order coherent scattering model which, relying on the Jones formalism, does not allow describing depolarizing phenomena.

The most powerful way to deal with polarimetric scattering from a distributed and depolarizing scene is to use the secondorder products of the scattering matrix [2].

Either an incoherent model, based on the Stokes formalism, or a coherent approach, based on the coherence $\mathbf{T}$ or covariance C matrices, can be used [1]. According to the Stokes formalism, the scattered field (described by the Stokes vector $s^{\mathrm{s}}$ ) is related to the incident field $s^{\mathrm{i}}$ by the $4 \times 4$ matrix $\mathbf{M}$, that is termed as Mueller matrix:

$$
\boldsymbol{s}^{\mathrm{s}}=(k r)^{-2} \mathbf{M} \boldsymbol{s}^{\mathrm{i}} .
$$

$\mathbf{M}$ is a real and never symmetric matrix whose elements are ensemble averages of combinations of the scattering amplitudes [3]. The elements of $\mathbf{M}$ are given by: [3]:

$$
\begin{gathered}
M_{11}=\frac{1}{2}\left\langle\left|S_{\mathrm{hh}}\right|^{2}+\left|S_{\mathrm{hv}}\right|^{2}+\left|S_{\mathrm{vh}}\right|^{2}+\left|S_{\mathrm{vv}}\right|^{2}\right\rangle, \\
M_{22}=\frac{1}{2}\left\langle\left|S_{\mathrm{hh}}\right|^{2}-\left|S_{\mathrm{hv}}\right|^{2}-\left|S_{\mathrm{vh}}\right|^{2}+\left|S_{\mathrm{vv}}\right|^{2}\right\rangle, \\
M_{33}=\left\langle\Re\left(S_{\mathrm{hh}} S_{\mathrm{vv}}{ }^{*}+S_{\mathrm{hv}} S_{\mathrm{hv}}{ }^{*}\right)\right\rangle, \\
M_{44}=\left\langle\Re\left(S_{\mathrm{hh}} S_{\mathrm{vv}}{ }^{*}-S_{\mathrm{hv}} S_{\mathrm{hb}}{ }^{*}\right)\right\rangle, \\
M_{12}=\frac{1}{2}\left\langle\left(\left|S_{\mathrm{hh}}\right|^{2}-\left|S_{\mathrm{vv}}\right|^{2}-\left|S_{\mathrm{hv}}\right|^{2}+\left|S_{\mathrm{vh}}\right|^{2}\right)\right\rangle, \\
M_{13}=\left\langle\Re\left(S_{\mathrm{hh}} S_{\mathrm{hv}}{ }^{*}+S_{\mathrm{vh}} S_{\mathrm{vv}}{ }^{*}\right)\right\rangle, \\
M_{14}=\left\langle\Im\left(S_{\mathrm{hh}} S_{\mathrm{hv}}{ }^{*}+S_{\mathrm{vh}} S_{\mathrm{vv}}{ }^{*}\right)\right\rangle, \\
M_{23}=\left\langle\Re\left(S_{\mathrm{hh}} S_{\mathrm{hv}}{ }^{*}-S_{\mathrm{hv}} S_{\mathrm{vv}}{ }^{*}\right)\right\rangle, \\
M_{24}=\left\langle\Im\left(S_{\mathrm{hh}} S_{\mathrm{hv}}{ }^{*}-S_{\mathrm{vh}} S_{\mathrm{vv}}{ }^{*}\right)\right\rangle, \\
\left.M_{34}=\left\langle\Im\left(S_{\mathrm{hh}} S_{\mathrm{vv}}{ }^{*}+S_{\mathrm{vh}}\right) S_{\mathrm{hv}}{ }^{*}\right)\right\rangle, \\
M_{21}=\frac{1}{2}\left\langle\left|S_{\mathrm{hh}}\right|^{2}+\left|S_{\mathrm{hv}}\right|^{2}-\left|S_{\mathrm{vh}}\right|^{2}{ }^{2}\left|S_{\mathrm{vv}}\right|^{2}\right\rangle, \\
M_{31}=\left\langle\Re\left(S_{\mathrm{hh}} S_{\mathrm{vv}}{ }^{*}-S_{\mathrm{hv}} S_{\mathrm{vv}}{ }^{*}\right)\right\rangle, \\
M_{32}=\left\langle\Re\left(S_{\mathrm{hh}} S_{\mathrm{vv}}{ }^{*}-S_{\mathrm{hv}} S_{\mathrm{vv}}{ }^{*}\right)\right\rangle, \\
M_{41}=\left\langle-\Im\left(S_{\mathrm{hh}} S_{\mathrm{vv}}{ }^{*}-S_{\mathrm{hv}} S_{\mathrm{vv}}{ }^{*}\right)\right\rangle, \\
M_{42}=\left\langle\Im\left(S_{\mathrm{hh}} S_{\mathrm{vv}}{ }^{*}-S_{\mathrm{hv}} S_{\mathrm{vv}}{ }^{*}\right)\right\rangle, \\
M_{43}=\left\langle-\Im\left(S_{\mathrm{hh}} S_{\mathrm{vv}}{ }^{*}+S_{\mathrm{hv}} S_{\mathrm{vh}}{ }^{*}\right)\right\rangle,
\end{gathered}
$$

where $\langle\cdot\rangle$ is the ensemble average operator, $*$ means complex conjugate and $\Re$ and $\Im$ stand for real and imaginary part, 
respectively. The Mueller matrix representation is the most general representation of polarimetric scattering [1], [2], and it has been demonstrated that there is a one-to-one mapping with other second-order scattering descriptors, i.e., $\mathbf{T}$ or $\mathbf{C}$. The elements in (4) refer to the most general bistatic case, simplifications apply when dealing with the monostatic configuration.

A second-order synthetic descriptor, widely used for classification purposes, is the co-polarized phase difference (CPD), i.e., the phase difference between the co-polarized scattering amplitudes [4]:

$$
C P D=\angle\left(S_{\mathrm{hh}} S_{\mathrm{vv}}{ }^{*}\right),
$$

The standard deviation (std) of the CPD has been found to be closely related to the randomness of the scattering mechanism [5]; with low (high) std being related to deterministic (random) mechanisms.

To detect metallic targets in a robust and effective way in [6] a metric was proposed that relies on the fact that, in the monostatic case, co- and cross-polarized channels are almost uncorrelated over natural scenarios due to reflection symmetry; while a non-negligible correlation applies when dealing with man-made metallic structures, e.g.; ships. This reasoning can be extended to the bistatic case where this symmetry condition implies that the correlation between co- and the sum between cross-polarized channels vanishes.

In this study, the capability of TanDEM-X to simultaneously collect imagery in both the monostatic (hereinafter active scene) and bistatic (hereinafter passive scene) configurations is exploited. The Mueller matrix and the CPD [7] are evaluated for both the active and passive scenes and results are intercompared.

\section{EXPERIMENTS}

In this section, some meaningful experiments related to the bistatic TanDEM-X acquisition of October 13rd, 2015 at 5:20 UTC in Gulf of Napoli, Napoli, Italy are presented and discussed. The incidence angle related to the center scene is $22.12^{\circ}$.

The 16 elements of the Mueller matrix related to the active scene are shown as graytone images in Fig. 1. The area under investigation, which includes both land and sea with some metallic targets most likely due to ships, is an excerpt of the whole SAR scene. The image related to the element $M_{11}$ is shown in the top-left corner of Fig. 1. This element is related to the SPAN and it clearly shows that both metallic targets and low backscatter areas are present in the area under investigation.

The same format is used to show the elements of the Mueller matrix related to an excerpt of the passive scene, see Fig. 2. It can be noted that differences apply between the two imagery that are mainly related to a different scattered intensity and a different weight played by cross-polarized channels. A zoomin version of the area under investigation (not shown to save space) shows that basically the scattered intensity related to the four channels is very similar for the active and passive scenes over large targets. Small targets tend to be less visible in the passive acquisition. This is likely due to the poorer signal-to-noise ratio that characterizes the passive scene.

The second experiments is related to the exploitation of the CPD, which is evaluated for both the active and the passive scenes. The histogram of the CPDs evaluated over a region of interest (ROI) related to free sea surface is shown in Fig. 3. It can be noted that, as expected, they both follow a Gaussian bell whose standard deviation is quite small (less that 30 degree) over sea surface. This witnesses that sea surface is responsible for a single-reflection Bragg scattering in both the active and passive scenes. On the other side, a Gaussian bell characterized by a larger standard deviation is obtained when evaluating the histogram over a low backscatter area or over urban areas (not shown to save space). Following this rationale, the CPD std is evaluated over the active and passive scenes using a $5 \times 5$ moving window. Results related to the active and passive acquisitions are shown in Fig. 4 (a) and (b), respectively.

The third experiment is related to the exploitation of reflection symmetry property to discriminate metallic targets from the surrounding sea. Since sea surface represents a natural distributes scenario, a negligible correlation between co- and cross-polarized channels is expected; while the latter increases significantly when dealing with metallic targets. The binary map obtained using a threshold equal to 0.1 is shown in Fig. 5(a). Note that land is also visible since the adopted metric is not normalized by the SPAN. The result obtained applying the metric $(h h(v h-h v))$ in the bistatic case is shown in Fig. 5(b). It can be noted that the bistatic case results in performance similar to the monostatic one.

\section{CONCLUSION}

In this study full-polarimetric TanDEM-X scenes collected simultaneously in monostatic and bistatic mode are exploited for maritime applications. The Mueller matrix and the standard deviation of the co-polarized phase difference are evaluated over a scene that includes sea, land and metallic targets. CPD std is shown to exhibit similar performance when evaluated using the active and passive scenes; while subtle differences apply when analyzing the terms of the Mueller matrix showing the different sensitivity of combination involving co- and cross-polarized channels in the monostatic and bistatic modes.

\section{ACKNOWLEDGMENT}

The authors would like to thank German Aerospace Center (DLR) for providing the data free of charge under the $\mathrm{AO}$ account NTI_POLI6644.

\section{REFERENCES}

[1] S.R. Cloude, "Polarisation: applications in remote sensing," Oxford University Press, 2009.

[2] J.J. van Zyl, C.H. Papas, and C. Elachi, "On the optimum polarization of incoherently reflected waves," IEEE Trans. Antennas Propag., vol. AP-35, no. 7, pp. 818-826, Jul. 1987.

[3] A. Guissard, "Mueller and Kennaugh matrices in radar polarimetry," IEEE Trans. Geosci and Remote Sens., vol. 32, no. 3, pp. 590-597, 1994. 

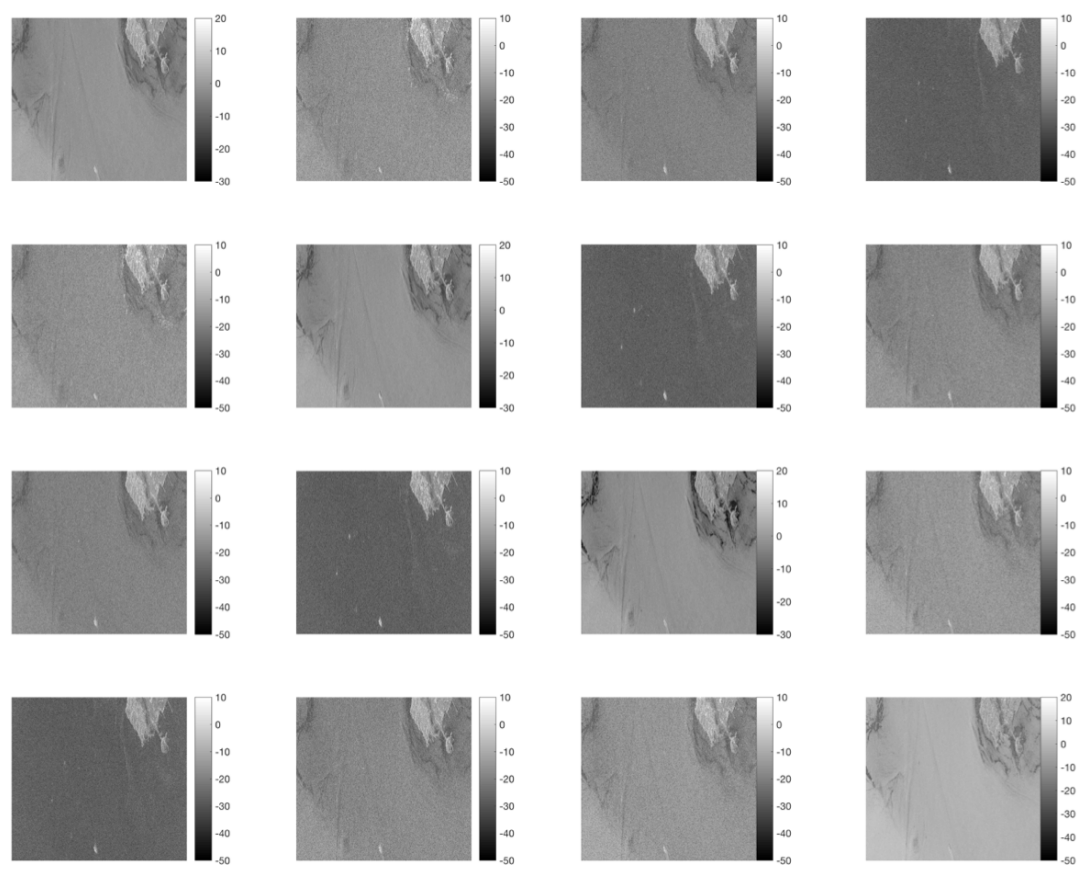

Fig. 1. Elements of the Mueller matrix, related to an excerpt of the scene observed by the active sensor, shown as grayscale images in $\mathrm{dB}$ units.
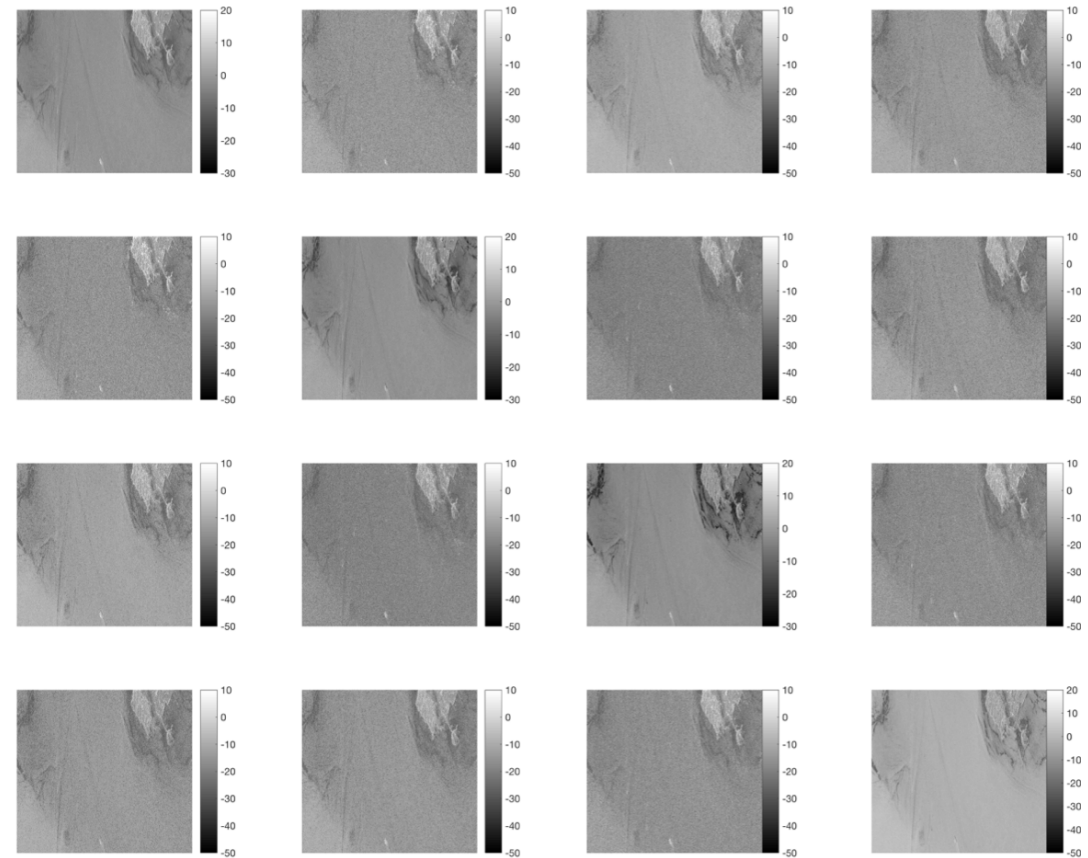

Fig. 2. Elements of the Mueller matrix, related to an excerpt of the scene observed by the passive sensor, shown as grayscale images in $\mathrm{dB}$ units.

[4] M. Migliaccio, A. Gambardella, F. Nunziata, M. Shimada, and O. Isoguchi, "The PALSAR polarimetric mode for sea oil slick observation," IEEE Trans. Geosci. Remote Sens., vol. 47, no. 12, pp. 4032-4041, Dec. 2009.

[5] D. Velotto, M. Migliaccio, F. Nunziata, and S. Lehner, "Dual-polarized TerraSAR-X data for oil-spill observation," IEEE Trans. Geosci. Remote Sens., vol. 49, no. 12, pp. 4751-4762, 2011.

[6] F. Nunziata, M. Migliaccio, and C.E. Brown, "Reflection symmetry for polarimetric observation of man-made metallic targets at sea," IEEE $J$. Oceanic Eng., pp. 384-394, 2012.

[7] F. Nunziata, A. Gambardella, and M. Migliaccio, "On the Mueller scattering matrix for SAR sea oil slick observation," IEEE Geosci. Remote Sens. Lett., vol. 5, no. 4, pp. 691-695, 2008. 


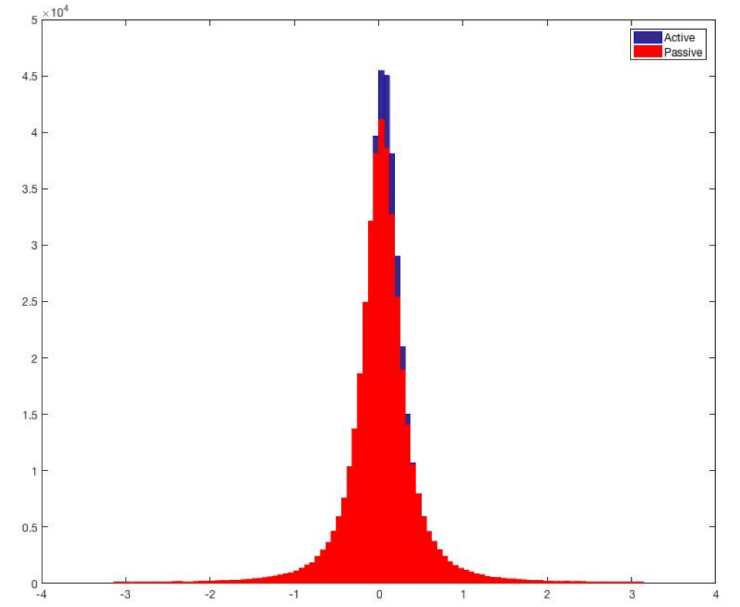

Fig. 3. Histograms of the CPD evaluated within a free sea surface ROI excerpted from the active (blue) and passive (red) acquisitions.

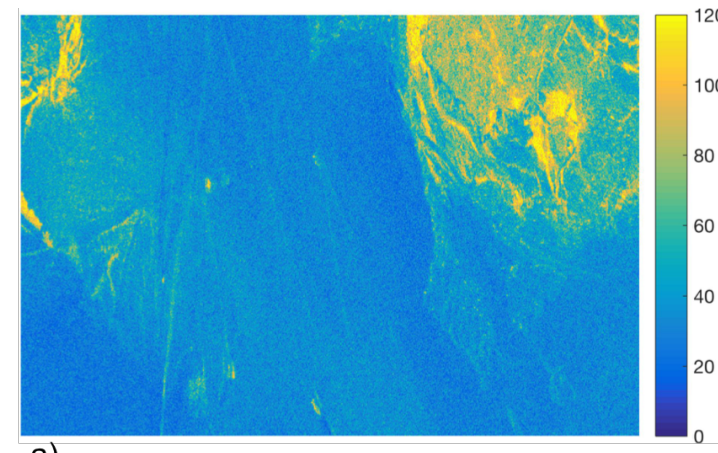

a)

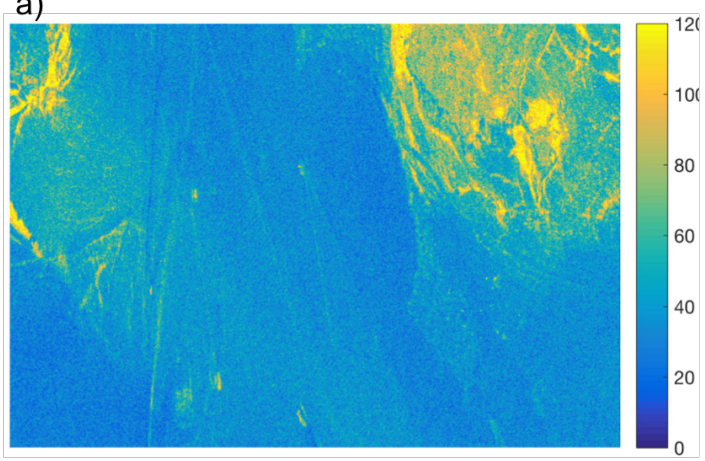

b)

Fig. 4. Standard deviation of the CPD evaluated over active (a) and passive (b) imagery using a $5 \times 5$ moving window.

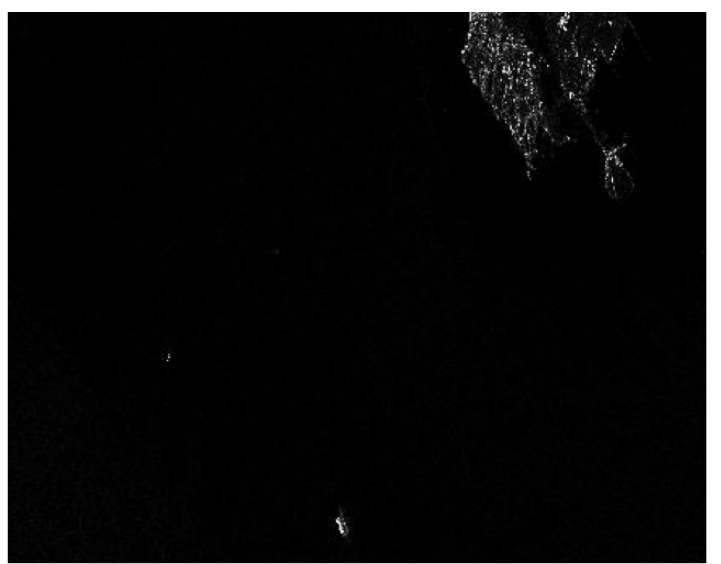

a)

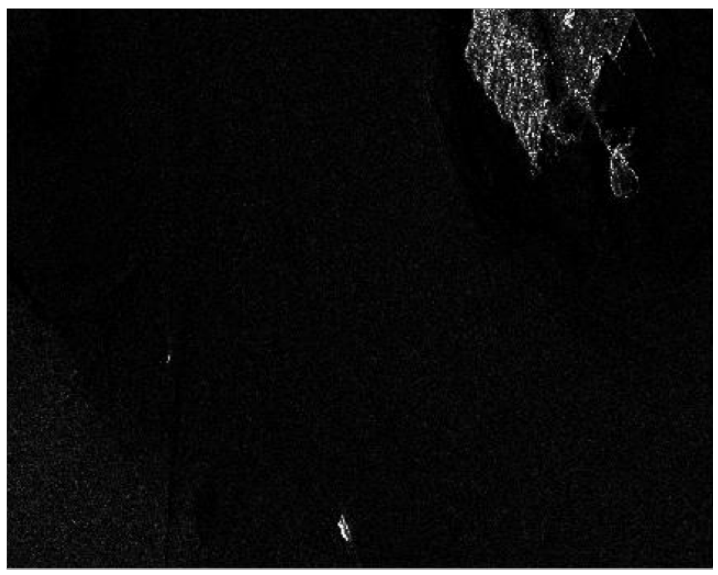

b)

Fig. 5. Reflection symmetry indexes related to the active (a) and passive scenes. Note that to obtain the binary images a threshold equal to .1 has been selected. 